\title{
An Unusual Case of Total Anomalous Pulmonary Venous Connection in Pregnancy
}

\author{
${ }^{1}$ Archana Kandasamy, ${ }^{2}$ Usha Vishwanath, ${ }^{3} \mathrm{M}$ Anitha, ${ }^{4}$ Priyanka Mehta
}

\begin{abstract}
Total anomalous pulmonary venous connection (TAPVC) is a rare congenital anomaly of the heart in which there is an absence of direct communication between the pulmonary veins and the left atrium, resulting in a mixture of oxygenated and deoxygenated blood in the right atrium. It is often associated with atrial septal defect or with transposition of great arteries, pulmonary atresia, truncus arteriosus, or single ventricle. The factors determining the severity are the severity of pulmonary venous obstruction and the restriction of interatrial communication. Uncorrected TAPVC rarely survives up to adulthood. Pregnancy in a patient with TAPVC is very rare unless surgically corrected in childhood. It often requires termination of pregnancy in first trimester as the heart cannot withstand the hemodynamic changes associated with pregnancy. We report a very rare case of pregnancy in a patient with uncorrected TAPVC, delivered successfully in our hospital without any complications.
\end{abstract}

Keywords: Congenital heart disease, Total anomalous pulmonary venous connection.

How to cite this article: Kandasamy A, Vishwanath $U$, Anitha M, Mehta P. An Unusual Case of Total Anomalous Pulmonary Venous Connection in Pregnancy. J South Asian Feder Obst Gynae 2017;9(2):190-191.

Source of support: Nil

Conflict of interest: None

Date of received: 11 January 2017

Date of acceptance: 16 March 2017

Date of publication: April 2017

\section{INTRODUCTION}

Total anomalous pulmonary venous connection (TAPVC) is a condition in which all the pulmonary veins have abnormal connection to the systemic circulation, wherein they do not connect directly to the left atrium. The reported incidence ranges from 0.4 to $2 \%$, which represents approximately seven persons in 1 lakh population. Often TAPVC occurs as an isolated lesion except for

\footnotetext{
${ }^{1}$ Postgraduate Student, ${ }^{2}$ Professor, ${ }^{3,4}$ Assistant Professor

${ }^{1-4}$ Department of Obstetrics and Gynecology, Sri Ramachandra University, Chennai, Tamil Nadu, India

Corresponding Author: Archana Kandasamy, Postgraduate Student, Department of Obstetrics and Gynecology, Sri Ramachandra University, Chennai, Tamil Nadu, India, Phone: +919884363939, e-mail: archanasrijay@gmail.com
}

association with atrial septal defect. Uncorrected TAPVC rarely survives up to adulthood. Pregnancy in a patient with TAPVC is very rare unless surgically corrected in childhood. It often requires termination of pregnancy in the first trimester itself as the heart cannot withstand the hemodynamic changes associated with pregnancy. We report a very rare case of pregnancy in a patient with uncorrected TAPVC, delivered successfully in our hospital without any complications.

\section{CASE REPORT}

A 29 years gravida 2 old lady, para 1, with previous intrauterine death had come to antenatal clinic at 8 weeks for regular antenatal care. Patient had spontaneous conception, confirmed by urine pregnancy test at 50 days of amenorrhea. There was no history of dyspnea, palpitations, or chest pain. She was a known case of heart disease since 1 year of age and was on treatment till 11 years of age, details of which were not available. There was no history of surgeries in the past. She belonged to lower socioeconomic class. Family history was insignificant. There were no reports or details available on previous pregnancy, intrauterine device at around 34 weeks, delivered vaginally.

Patient was admitted and evaluated. O/E patient was comfortable at rest, afebrile, with blood pressure of $120 / 70 \mathrm{~mm} \mathrm{Hg}$, pulse rate 82 / minute, saturation of $99 \%$ in room air. Cardiovascular system - S1 and S2 heard, pansystolic murmur heard throughout, single S2 and A2 audible at left cardiac border. Respiratory sound - normal vesicular breathe sounds heard. Hemoglobin was 15.2 gm $\%$, total white blood cell count $13,200 \mathrm{~mm}^{3}$, fasting and postprandial sugars 80 and $76 \mathrm{mg} / \mathrm{dL}$ respectively. Blood group was A positive. Screening echo done showed right ventricular dysfunction, ejection fraction (EF) of $60 \%$, with severe pulmonary valve stenosis and mild pulmonary regurgitation. Cardiology opinion sought and advised to continue pregnancy with plan of balloon pulmonary valvuloplasty at 28 weeks. Viability scan showed single live intrauterine gestation at 8 weeks and 3 days. The patient was asymptomatic and discharged and followed every 2 weeks. First trimester screening showed low risk for Down's, Edward's, and Patau syndromes. Anomaly scan was done at 20 weeks and no gross anomalies were detected. In view of placental lakes, magnetic resonance 


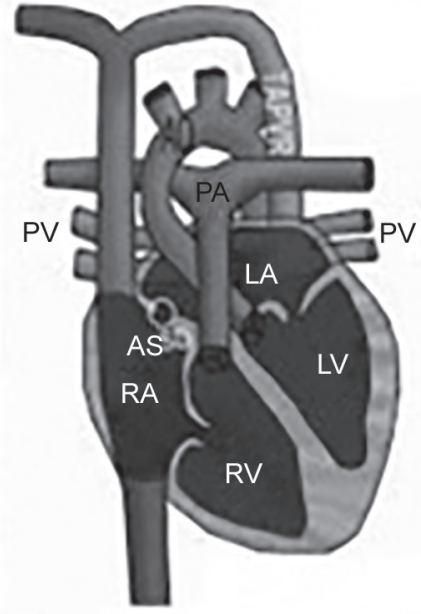

Fig. 1: Total anomalous pulmonary venous connection showing abnormal connection

imaging (MRI) was done, which showed heterogeneous areas of chronic and subacute hemorrhage. Fetal echo was normal.

She was admitted at 26 weeks for biophysical profile. Ultrasound showed $844 \mathrm{gm}$ live fetus with posterior placenta and liquor lower limit of normal. Echo showed mild mitral regurgitation, normal left ventricular systolic function, EF $60 \%$. At 27 weeks, in view of cyanosis and clubbing, cardiologist opinion was sought. Cardiac MRI done confirmed TAPVC with atrial septal defect and pulmonary stenosis and advised for termination of pregnancy and corrective cardiac surgery or continuation of pregnancy under high risk of life (Fig. 1). Hence, neonatologist counseling was given. Patient and family opted to continue pregnancy under high risk and highrisk consent was obtained. Repeat biophysical profile at 29 weeks showed fetus corresponding to 29 weeks, estimated fetal weight of $1.2 \mathrm{~kg}$, amniotic fluid index $17.3 \mathrm{~cm}$, with features of intrauterine growth restriction (IUGR) (abdominal circumference at 20th centile, femur length at 10th centile). Umbilical artery Doppler showed high resistance flow and fetal middle cerebral artery (MCA) showed brain sparing effect. Repeat Doppler at 29 weeks +1 day showed umbilical artery absent diastolic flow, fetal MCA increased diastolic flow, ductus venosus normal - suggestive of cerebral redistribution. Neonatologist, cardiologist, anesthetist team called in and planned for emergency lower segment cesarean section under moderate risk in view of IUGR with Doppler changes. Patient delivered a boy baby of $1.05 \mathrm{~kg}$, with APGAR score $8 / 10,9 / 10$.

There was no intraoperative complications. Postoperatively, patient was put in intensive care unit (ICU) with ventilatory support. Patient had one episode of general tonic-clonic seizures on postoperative day 1 , which was conservatively managed. The MRI brain done was normal. Patient was extubated on postoperative day 2 .
No further complications were noted. Baby was under preterm care in neonatal ICU. Patient recovered well without further complications. Mother and baby were discharged after 2 months of preterm care.

\section{DISCUSSION}

The TAPVC connection is a rare congenital anomaly of the heart in which there is an absence of direct communication between the pulmonary veins and the left atrium, resulting in a mixture of oxygenated and deoxygenated blood in the right atrium. ${ }^{3}$ It is often associated with atrial septal defect or with transposition of great arteries, pulmonary atresia, truncus arteriosus, or single ventricle. It is classified based on anatomical location of the anomalous connection as supracardiac, cardiac, infracardiac, and mixed. The factors determining the severity are the severity of pulmonary venous obstruction and the restriction of interatrial communication. Usually, neonates with TAPVC present with cyanosis and tachypnea within 48 hours of birth and others are detected at 1 year of age. Chest X-ray, echo, and MRI ${ }^{2}$ help in diagnosing the condition, definitive being cardiac catheterization. Uncorrected TAPVCs do not survive till adulthood.

Pregnancy in patients with TAPVC is extremely rare and often an indication for termination of pregnancy since hemodynamic changes in pregnancy, ${ }^{4}$ such as increased cardiac output and heart rate are not tolerated in patients with TAPVC. Present case here survived up to adulthood and delivered successfully at our institution. Patient had TAPVC associated with atrial septal defect and pulmonary stenosis and hence, most of the oxygenated blood could reach the left atrium and the systemic connection and is thus compatible with life. Patient was discharged with the baby and advised to follow up for corrective surgery. Multidisciplinary team approach helped in a successful management and delivery without complications in our tertiary care hospital.

\section{REFERENCES}

1. Webb, GD.; Smallhorn, JF.; Therrien, J.; Redington. AN. Congenital heart disease. In: Bonow RO, Mann DL, Zipes DP, Libby P, editors. Braunwald's heart disease: a textbook of cardiovascular medicine. 9th ed. Chap 65. Philadelphia, PA: Saunders Elsevier; 2011.

2. Prasad SK, Soukias N, Hornung T, Khan M, Pennell DJ, Gatzoulis MA, Mohiaddin RH. Role of magnetic resonance angiography in the diagnosis of major aortopulmonary collateral arteries and partial anomalous pulmonary venous drainage. Circulation 2004 Jan;109(2):207-214.

3. Darling RC, Rothney WB, Craig JM. Total pulmonary venous drainage into the right side of the heart: report of 17 autopsied cases not associated with other major cardiovascular anomalies. Lab Invest 1957 Jan-Feb;6(1):44-55.

4. Bangal VB, Gupta K, Gavhane SP, Gangapurwala S. Rare case of pregnancy with total anomalous pulmonary venous connection (TAPVC). Int J Biomed Res 2014 Feb;5(2):143-144. 